DTP $/ 93 / 64$

August 1993

\title{
How suppressed are the radiative interference effects in heavy unstable particle production?
}

\author{
V.S. Fadin', V.A. Khoze and A.D. Martin \\ Department of Physics, University of Durham, \\ Durham DH1 3LE, England.
}

\begin{abstract}
We present a "theorem" which quantifies, for inclusive processes, the level of suppression of the radiative interference effects between the production and decay stages of heavy unstable particles. The theorem, which is based on very general physical arguments, is applicable to all orders in the coupling.
\end{abstract}

\footnotetext{
${ }^{1}$ Permanent address: Budker Institute for Nuclear Physics and Novosibirsk State University, 630090 Novosibirsk, Russia.
} 
The study of heavy unstable particles (e.g. $t$ quark or $W$ boson) is of particular interest in high energy physics. Since the typical width of such a particle is large, $\Gamma \sim O(1 \mathrm{GeV})$, the particle is not observed itself, but rather it is identified by its decay products. Therefore in production processes of heavy unstable particles it is natural to separate the production stage from the decay processes. In general these stages are not independent and may be interconnected by radiative interference effects. Particle(s) (e.g. photon(s) and/or gluon(s)) could be produced at one stage and absorbed at another; we speak of virtual interference. Real interference will occur as well since the same real particle can be emitted from the different stages of the process.

Many observations rely on a clear understanding of the role of these interference effects. Indeed there is a long list of examples where a detailed knowledge of the effects can be important for the interpretation of experimental data (see, for example, refs. [1, 2]). For instance, the interference phenomena have to be quantified to obtain the complete $O\left(\alpha_{s}\right)$ corrections to $e^{+} e^{-} \rightarrow t \bar{t} \rightarrow W^{+} W^{-} b \bar{b}$ and the $O\left(\alpha_{s}^{2}\right)$ corrections to $e^{+} e^{-} \rightarrow W^{+} W^{-} \rightarrow(4$ jets $)$ inclusive cross sections. (Note that the leading QCD interference correction to the latter process is $O\left(\alpha_{s}^{2}\right)$ to ensure colour conservation.) Clearly such corrections are relevant, interalia, for an accurate experimental determination of heavy particle masses.

In ref. [1] we made an explicit study, with the help of soft-insertion techniques, of the interference phenomena between the production and decay stages to $O\left(\alpha_{\text {int }}\right)$ (where $\alpha_{\text {int }}=\alpha$ or $\alpha_{s}$ as appropriate) and, in each case, we identified the particular degree of inclusiveness of the process that is required for the interference effects to be suppressed. Of course, the real and virtual interference contributions, taken separately, are not suppressed but are infrared divergent. Clearly the infrared divergent parts have to cancel for physically meaning values. This is not the issue. Rather the crucial question is to what level does this cancellation occur?

Here we present a general theorem which states that the effects of radiative interferences (between the various production and decay stages) are each suppressed by $O(\Gamma / M)$ in the totally inclusive production of unstable particles. Our proof does not rely on specific assumptions, like soft-insertion factorization, and the resulting theorem is applicable to any order in the coupling $\alpha_{\text {int }}$.

Before presenting the proof it may be useful to express the resulting recipe in a symbolic form. To be specific let us consider the production of $N$ heavy unstable particles $A_{1}, \ldots A_{N}$ with masses $M_{i}$ and widths $\Gamma_{i}$. For reference purposes we first consider the production in the 
absence of the decays of the heavy particles (i.e. we switch off the interaction responsible for the decays). Then the inclusive cross section may be written in the form

$$
\sigma_{\text {stable }}\left(A_{1}, \ldots A_{N}\right)=\sigma_{0}\left(M_{i}^{2}\right)(1+\delta(R, C))
$$

where $\sigma_{0}\left(M_{i}^{2}\right)$ is the production cross section in the Born approximation and $\delta(R, C)$ represents the radiative corrections. Here we have separated possible Coulombic corrections $C$ from the remaining radiative corrections $R$. The Coulombic effects $C$, which are associated with large space-time intervals, are only important [3] if two charged (or coloured) particles are slowly movingf in their c.m. frame (e.g. $W^{+} W^{-}$or $t \bar{t}$ production near threshold). Now the question is "how is (1) modified in the physical case when we allow the heavy unstable particles $A_{i}$ to decay?" We will show

$$
\sigma_{\text {unstable }}\left(A_{1}, \ldots A_{N}\right)=\int \prod_{i}\left(d s_{i} \rho\left(s_{i}\right)\right) \sigma_{0}\left(s_{i}\right)(1+\delta(R, \bar{C}))+\sum_{n} O\left(\alpha_{\text {int }}^{n} \frac{\Gamma_{i}}{M_{i}}\right)
$$

with

$$
\rho\left(s_{i}\right)=\frac{\sqrt{s_{i}} \Gamma_{i}\left(s_{i}\right)}{\pi\left[\left(s_{i}-M_{i}^{2}\right)^{2}+s_{i} \Gamma_{i}^{2}\left(s_{i}\right)\right]}
$$

where $\Gamma_{i}\left(s_{i}\right)$ is "running" physical width which incorporates the radiative effects associated solely with the decay of particle $A_{i}$.

In other words, the theorem for the production of heavy unstable particles says that, apart from the two modifications explicitly shown in the formula, the introduction of the particle widths gives rise to no new corrections up to order $\alpha_{\mathrm{int}}^{n} \Gamma_{i} / M_{i}$, where the $n=0$ term corresponds to the standard (non-radiative) non-resonant backgrounds and the $n \geq 1$ terms to interference induced by $n$ radiated quanta. The two modifications in going from (1) to (2) are, first, the natural kinematic effect leading to the integrations over $\rho\left(s_{i}\right)$ and, second, the modification (symbolically shown as $C \rightarrow \bar{C}$ ) of the Coulombic interaction between particle pairs which are non-relativistic in their c.m. frame [4, 5]. In particular, the theorem says the remaining radiative corrections are unchanged.

The proof of the theorem relies on two facts. First, the suppression (by at least a factor $\left.\Gamma /\left|k^{0}\right|\right)$ of interferences between the various production and decay stages arising from energetic

\footnotetext{
${ }^{2}$ Of course the separation of Coulomb effects can only be done uniquely near threshold, but this is the very region where the instability effects are most important.

${ }^{3}$ Strictly speaking the notation $\sigma\left(A_{1}, \ldots A_{N}\right)$ in formula $(2)$ is imprecise in the sense that the same final state may be reached without going through resonances $A_{1}, \ldots A_{N}$.
} 
photons/gluons with $\left|k^{0}\right| \gg \Gamma$, and, second, the absence of infrared divergences in the total or inclusive cross-section. Both facts have a simple physical interpretation. Let us consider, without loss of generality, the case when the total energy of the process is of the order of the masses $M_{i} \sim M$ of the produced unstable particles. Then the typical time, $\tau_{p}$, of the duration of the production stage, as well as of the decay stages, is of order of $1 / M$. This time $\tau_{p}$ is much less than the characteristic time $\Delta t$ between the various stages; since the typical time between the production and decay stages of any heavy particle $i$ is $\Delta t=\Delta t_{i} \sim 1 / \Gamma_{i} \sim 1 / \Gamma$, and that between the decay stages of heavy particles $i$ and $j$ is

$$
\Delta t=\Delta t_{i j} \sim \max \left(\frac{1}{\Gamma_{i}}, \frac{1}{\Gamma_{j}}\right) \sim \frac{1}{\Gamma} .
$$

As a consequence the relative phases of photon/gluon emissions (or between emission and absorption) at the different stages of the process are approximately equal to $\left|k^{0}\right| \Delta t \sim\left|k^{0}\right| / \Gamma$. When $\left|k^{0}\right| \gg \Gamma$ this phase shift is large and therefore the radiative interference effects are suppressed.

This physical picture is readily confirmed by inspection of the propagators of the unstable particles. Photon/gluon emission with energy $\left|k^{0}\right| \gg \Gamma$ shifts the invariant mass of the unstable particle far from its resonant value, so for an interference contribution we obtain a factor $\sim 1 /(i \Gamma M)\left(k^{0} M-i \Gamma M\right)$, instead of the factor $\sim|1 / i \Gamma M|^{2}$ in the absence of radiation. Thus we have suppression of hard photon/gluon interference effects by at least a factor of $\Gamma /\left|k^{0}\right|$.

The second basic fact, the absence of infrared divergences in totally inclusive cross-sections [6], has also a clear physical interpretation. Infrared divergences appear when we use states containing a definite number of photons/gluons. Now the acceleration of charge/colour leads to radiation with finite spectral intensity at zero frequency and so the scattering or the creation of charged/coloured particles is accompanied by the emission of an infinite number of photons/gluons. To obtain a physically meaningful cross section (which can be expanded in powers of $\alpha$ or $\alpha_{s}$ ) we must include arbitrary numbers of emitted photons or gluons. In the case of gluons we need also to average over the initial colour states, because colour is changed in the process of gluon emission.

For the proof we only need the absence of those infrared divergences in the total cross-section which are connected with the radiative interference between the various production and decay stages of the process. To show this we first note that the total cross-section is proportional to the imaginary part of the forward scattering amplitude. When this amplitude is expressed 
as the sum of Feynman diagrams, all particles except the initial particles, appear as internal lines, that is they correspond to virtual particles. Therefore the interference photon/gluon lines must be attached to internal lines, at least at one end. But it is well known (and can be readily verified by simple power counting) that infrared divergent contributions only arise from photons/gluons with lines which couple at both ends to external lines corresponding to on-mass-shell particles. Because of the absence of infrared divergences the contribution from the region of small photon/gluon energies $\left|k^{0}\right| \lesssim \Gamma$ is small due to the lack of phase space. On the other hand, as shown above, for $\left|k^{0}\right| \gg \Gamma$ the interference effects are small due to the large time separations between the various stages. Therefore radiative interference between the production and decay stages is suppressed by at least a factor $\Gamma / M$.

So far we have considered interference induced by a single photon or gluon. For multiple exchanges the situation is slightly different but the conclusion remains valid. In the case of multiple exchange large energies $k_{i}^{0}$ of individual photons/gluons are allowed, since the constraint that the invariant mass of the unstable particle must not be shifted far from its resonant value only requires

$$
\left|\sum_{i} k_{i}^{0}\right| \lesssim \Gamma
$$

where the sum is performed over photons or gluons emitted $\left(k_{i}^{0}>0\right)$ and absorbed $\left(k_{i}^{0}<0\right)$ at one of the decay stages. But because of the absence of infrared divergences there is again a suppression of at least one factor of $\Gamma / M$ due to the restriction of the phase space imposed by condition (4).

Finally we must consider the Coulomb radiative effects. Now there are infrared singularities connected with the Coulomb interaction of charged/coloured particles which are special in the sense that they are not cancelled by real emissions (which have only transverse polarization states), but rather they appear in matrix elements as a phase factor with an infinite phase. Therefore they do not appear in the expression for the cross section and are not seen at all in our approach where we express the cross section in terms of the forward scattering amplitude.

However there is a Coulomb interaction which is connected with small photon/gluon frequencies, but which is not infrared divergent. For two charged/coloured particles, with reduced mass $\mu$ and momentum $\boldsymbol{q}$ in their c.m. frame, the essential energies $k_{c}^{0}$ of the exchanged Coulombic photons/gluons are typically $\left|k_{c}^{0}\right| \sim \boldsymbol{q}^{2} / \sqrt{\left(\boldsymbol{q}^{2}+\mu^{2}\right)}$. When $\boldsymbol{q}^{2} \lesssim \mu \Gamma$ these energies are $\left|k_{c}^{0}\right| \lesssim \Gamma$. Thus for two slowly moving charged/coloured particles in their c.m. frame there is an important Coulombic radiative interaction coming from the region of small photon/gluon energies. 
At first sight this appears to violate our previous statement, that the contribution from the region $\left|k^{0}\right| \lesssim \Gamma$ is small. But that statement referred to interference photons/gluons and it remains correct for them. The reason is that for interference between the different production and decay stages of a process, the only important Coulomb interactions are those between a charged/coloured decay product of one of the unstable particles and some other particle (e.g. another unstable particle or one of its decay products) with the interacting pair slowly moving in their c.m. frame (that is $\boldsymbol{q}^{2} \lesssim \mu \Gamma$ ). This corresponds to a very small region of the available phase space and so these Coulomb effects are also suppressed by at least a factor $\Gamma / M$. This concludes the proof of the theorem.

Some words should be added to explain the modification $\delta(R, C) \rightarrow \delta(R, \bar{C})$ in going from formula (1) for "stable" heavy particles to the realistic formula (2) for unstable particles. Away from the heavy particle production threshold the typical heavy particle interaction time is $1 / \sqrt{s} \lesssim 1 / M$, i.e. much smaller than their lifetimes. Thus the influence of instability on the Coulomb corrections at the production stage gives effects of relative order $\Gamma / M$ or less. Thus $\delta(R, \bar{C}) \approx \delta(R, C)$.

The situation is different for heavy unstable particle production near threshold. Then the typical Coulomb interaction time $\tau_{c}$ can be comparable to, or even larger than, the particle's lifetime $\tau$

$$
\tau_{c} \sim \frac{1}{k_{c}^{0}} \sim \frac{\mu}{\boldsymbol{q}^{2}} \gtrsim \frac{1}{\Gamma}=\tau .
$$

Therefore the Coulomb part $C$ of the radiative correction $\delta$ shown in (1) will be considerably modified by instability, and hence it is denoted by $\bar{C}$ in (2). The calculation [4, 回 of the modified contribution $\bar{C}$ can be best done using old-fashioned non-relativistic perturbation theory, particularly as these effects are only crucial near threshold. The diagrams are the same as in the stable particle case, but we require the momentum $p_{i}=\left(\epsilon_{i}, \boldsymbol{p}_{i}\right)$ of $A_{i}$ to satisfy $p_{i}^{2}=s_{i}$, and we must replace the energies of the unstable particles by

$$
\epsilon \longrightarrow \epsilon+\frac{i M \Gamma}{2 \sqrt{p^{2}+M^{2}}}
$$

in the energy denominators of all intermediate states.

In conclusion, formula (2) gives a transparent recipe for quantifying the level of suppression of the radiative interference effects (which occur between the production and decay stages) in the inclusive production of heavy unstable particles. Moreover we detail how the modifications due to instability may be implemented (i.e. the introduction of $\rho\left(s_{i}\right)$ containing the physical 
width $\Gamma\left(s_{i}\right)$ and the modifications $C \rightarrow \bar{C}$ of the Coulomb effects near threshold).

\section{Acknowledgements}

This work was supported in part by the U.K. Science and Engineering Research Council. V.S.F. thanks the Centre for Particle Theory at the University of Durham for hospitality. V.A.K. is grateful to Yu.L. Dokshitzer for useful discussions.

\section{References}

[1] V.S. Fadin, V.A. Khoze and A.D. Martin, Durham preprint DTP/93/18 (1993).

[2] Yu.L. Dokshitzer, V.A. Khoze, L.H. Orr and W.J. Stirling, Nucl. Phys. B403 (1993) 65.

[3] A. Sommerfeld, "Atombau und Spektrallinien", Bd. 2 (Vieweg, Braunschweig, 1939); A.D. Sakharov, JETP 18 (1948) 631.

[4] V.S. Fadin and V.A. Khoze, JETP Letters 46 (1987) 525.

[5] V.S. Fadin, V.A. Khoze and A.D. Martin, Phys. Lett. B311 (1993) 311.

[6] F. Bloch and A. Nordsieck, Phys. Rev. 52 (1937) 54;

D.R. Yennie, S.C. Frautschi and H. Suura, Ann. Phys. (NY) 13 (1961) 379;

T. Kinoshita, J. Math. Phys. 3 (1962) 650;

T.D. Lee and M. Nauenberg, Phys. Rev. 133 (1964) B1549. 